\title{
Improving Multidrug-Resistance Tuberculosis Papua's Management Using Whole Genome Sequencing
}

\author{
Yustinus Maladan $^{1^{*}}$, Hana Krismawati ${ }^{1}$, Antonius Oktavian ${ }^{1}$, C. S Whinie Lestari ${ }^{2}$ \\ ${ }^{1}$ Center for Health Research and Development, Jayapura, Indonesia \\ ${ }^{2}$ Center for Research and Development of Biomedical and Basic Health Technology, Jakarta, Indonesia \\ *Corresponding author. Email: justin.maladan@gamil.com
}

\begin{abstract}
One of the challenges in TB control of Papua is increasing number of multidrug-resistance tuberculosis (MDR-TB). The aim of the study is to imply the WGS tools for predicting TB drugs resistance even MDR as well XDR in clinical samples as an initiation model of laboratory improvement for accurate treatment in MDR TB. Twenty Lowenstein Jansen M. tuberculosis cultures from sputum clinical samples were taken as a DNA source for WGS. Samples preparation was perform using Nextera XT Kit by Illumina and read by MiSeq whole genome sequencing. Sequencing result was analyze using TB profiler and visualize by UGENE. We found mutations that direct to drugs resistance in 19 samples consist of 14 samples are MDR and 5 samples are non MDR mutation. Among the 4 samples of non MDR, 2 of them previously show mutation of rifampicin resistance in gene expert but non mutation resistance in WGS. Whole Genome Sequencing technology is the advance technology that may apply to predict the resistance of TB drugs even MDR or XDR and distinguish the accurate drugs for patients individually.
\end{abstract}

Keywords: stochastic MDR-TB, tuberculosis, whole genome sequencing, rifampicin

\section{INTRODUCTION}

Recently, Indonesia is still considered as the 10 top rank countries for Tuberculosis (TB). Based on the Case Detection Rate, the provinces with the highest CDR were DKI Jakarta (122.2\%), South Sulawesi (84.0\%), Papua (78.5\%) [1]. An increasing number of HIV / AIDS in the world adds to the problem of TB including in Indonesia especially Papua, become the new burden in TB elimination since coinfection with HIV will significantly increase the risk of Mycobacterium tuberculosis infection. Besides that, the emerging drug resistance in tuberculosis becomes a challenge in TB treatment and control [2].

Improving biomolecular technology, open many possibilities to conduct a genomic study that can predict the presence of mutations associated with resistance to OAT both first and second line before therapy of $\mathrm{M}$. tuberculosis. The successful diagnosis and treatment of $\mathrm{M}$ / XDR-TB depend on universal access to accurate drugsusceptibility testing (DST). Conventionally, the diagnosis of drug resistance in M. tuberculosis (MTB) takes high and laboratory effort since the slow culture of $\mathrm{M}$. tuberculosis and biosafety level 3 facility necessities. However, phenotypic results are only obtained after weeks of months of incubation, and many countries lack the resources to establish the stringent laboratory conditions required for these growth-based testing methods $[3,4]$.

\begin{abstract}
Next-generation sequencing (NGS) is a powerful tool in the detection of all clinically relevant mutations, and thereby the rapid diagnosis of drug-resistant TB (DR-TB) in clinical specimens [3,5]. The accumulation of WGS data allows us to assess the genetic diversity across the genome, seeking signatures of selective pressure [5]. Many countries apply WGS for detecting MDR and XDR Tuberculosis [6-13]. Whole-genome sequencing of bacteria has been shown to provide comprehensive data like as prediction of drug susceptibility and resistance, epidemiological analysis and research [14-16]. WGS has the potential to revolutionize the definition of drug susceptibility testing (DST) of MTB in both high and lowincome settings, and a growing knowledge of the genetic mechanisms of resistance, combined with an improved IT infrastructure, will facilitate its adoption and enhance its clinical utility for drug testing [14].
\end{abstract}

Implementation of this WGS assay on all cases of MTBC has aided TB control efforts and improved the accuracy of molecular resistance predictions being reported to physicians, resulting in more effective patient management and drug resistance surveillance such as New York State 
samples are successfully sequenced properly and one of them could not be analyzed. Sequencing data is obtained in FASQ format. The data is analyzed using TB Profiler. Output from TB profile obtained comprehensive data on the profile of gene mutations associated with TB drug resistance in both the first-line drug group and the secondline drug group (Table 1).

\section{METHOD}

\section{Samples}

This was a cross-sectional study taking place in Jayapura. Samples were obtained from patients suspected of tuberculosis in Jayapura BSL level 3 Regional Health Laboratory. Initial identification of drug resistance was carried out using GeneXpert. Samples that were positively resistant in geneXpert were cultured on LowensteinJensen's media. Twenty samples of M. tuberculosis culture were extracted their DNA using DNA mini-column Qiagen Kit.

\section{Library Preparation and Sequencing}

The quality was assessed by fluorometric quantification, Qubit $^{\text {TM }}$ 3.0 Fluorometer with a dsDNA Broad Range Assay Kit (Thermo Fisher Scientific) and agarose gel electrophoresis. Prosedur Next Generation Sequencing followed of protocol Nextera XT DNA Library Prep Kit. NGS was performed per manufacturer's instructions (Illumina, San Diego, CA, USA) using MiSeq 600 cycle Reagent Kit (V3). Whole Genome Sequencing of M. tuberculosis was carried out using the Next Generation Sequencing method on the MiSeq machine by following the steps as follows: Tagging of M. tuberculosis Genome using Nextera transposome. Library amplification was performed using Nextera PCR Master Mix. The PCR results are then cleaned with AMPure XP beads. After cleaning, the library was normalized with the Nextera XT DNA Library Preparation Kits. Normalized DNA was pooled in a single tube then diluted by following the Protocol Dilution libraries using the Dilute Libraries Guide Protocol B: BeadBased Normalization Method. After that it was put into the cartridges and running on MiSeq. Libraries were sequenced with an Illumina MiSeq V3 and 300-bp paired-end reads with samples randomized across two runs (each $\sim 24 \mathrm{~h}$ in duration).

\section{Bioinformatics Pipeline}

Genome analysis was perform using software TB profiler (https://github.com/jodyphelan/TBProfiler) 17 on platform Linux 17.10. BAM file Analyzing, reading visualization, creating consensus and alignment were done using Unipro Ugene NGS 1.3.1.18.

\section{RESULTS AND DISCUSSION}

A total of 20 clinical samples from TB patients in Papua have been downloaded using the NGS method. Nineteen 
Table 1. Profile Of Drugs Resistance TB Of M. Tuberculosis Strain From Papua Using TBProfiler

\begin{tabular}{|c|c|c|c|c|c|c|c|c|c|c|c|c|c|c|c|c|c|c|}
\hline \multirow{3}{*}{ Sample } & \multirow{3}{*}{$\begin{array}{l}\text { Gene } \\
\text { Xpert }\end{array}$} & \multicolumn{16}{|c|}{ Whole Genome Sequencing } & \multirow{3}{*}{ Note } \\
\hline & & \multicolumn{5}{|c|}{ Group 1: First line agents } & \multicolumn{3}{|c|}{$\begin{array}{l}\text { Group 2: Injectable } \\
\text { agents }\end{array}$} & \multicolumn{3}{|c|}{$\begin{array}{l}\text { Group 3: Gol. } \\
\text { Floroquinolone }\end{array}$} & \multicolumn{5}{|c|}{$\begin{array}{c}\text { Group 4: Oral bacteriostatic second line } \\
\text { agents }\end{array}$} & \\
\hline & & $\mathbf{H}$ & $\mathbf{R}$ & $\mathbf{E}$ & $\mathbf{Z}$ & $\mathbf{S}$ & $\mathbf{K m}$ & $\mathbf{A m}$ & $\mathbf{C m}$ & Ofx & Lfx & Mfx & Eto & Pto & Cs & PAS & Trd & \\
\hline TB0065 & $\mathrm{R}$ & $\mathrm{R}$ & $\mathrm{R}$ & $\mathrm{R}$ & - & $\mathrm{R}$ & - & - & - & - & - & - & - & - & - & - & - & MDR \\
\hline TB0207 & $\mathrm{R}$ & $\mathrm{R}$ & $\mathrm{R}$ & - & - & - & - & - & - & - & - & - & $\mathrm{R}$ & - & - & - & - & MDR \\
\hline TB035 & $\mathrm{R}$ & $\mathrm{R}$ & $\mathrm{R}$ & $\mathrm{R}$ & - & $\mathrm{R}$ & - & - & - & - & - & - & - & - & - & - & - & MDR \\
\hline TB062 & $\mathrm{R}$ & $\mathrm{R}$ & $\mathrm{R}$ & - & - & - & - & - & - & - & - & - & - & - & - & - & - & MDR \\
\hline TB1007 & $\mathrm{R}$ & $\mathrm{R}$ & $\mathrm{R}$ & - & - & - & - & - & - & - & - & - & - & - & - & - & - & MDR \\
\hline TB1023 & $\mathrm{R}$ & - & $\mathrm{R}$ & - & - & - & - & - & - & - & - & - & - & - & - & - & - & - \\
\hline TB1186 & $\mathrm{R}$ & $\mathrm{R}$ & $\mathrm{R}$ & - & - & - & - & - & - & - & - & - & - & - & - & - & - & MDR \\
\hline TB240 & $\mathrm{R}$ & - & $\mathrm{R}$ & - & - & - & - & - & - & - & - & - & - & - & - & - & - & - \\
\hline TB284 & $\mathrm{R}$ & $\mathrm{R}$ & $\mathrm{R}$ & $\mathrm{R}$ & $\mathrm{R}$ & - & - & - & - & - & - & - & - & - & - & - & - & MDR \\
\hline TB345 & $\mathrm{R}$ & $\mathrm{R}$ & $\mathrm{R}$ & - & - & - & - & - & - & - & - & - & - & - & - & - & - & MDR \\
\hline TB487 & $\mathrm{R}$ & $\mathrm{R}$ & $\mathrm{R}$ & - & - & - & - & - & - & - & - & - & - & - & - & - & - & MDR \\
\hline TB524 & $\mathrm{R}$ & - & $\mathrm{R}$ & - & - & - & - & - & - & - & - & - & - & - & - & - & - & - \\
\hline TB530 & $\mathrm{R}$ & $\mathrm{R}$ & $\mathrm{R}$ & $\mathrm{R}$ & - & - & - & - & - & - & - & - & - & - & - & - & - & MDR \\
\hline TB618 & $\mathrm{R}$ & - & - & - & - & $\mathrm{R}$ & $\mathrm{R}$ & $\mathrm{R}$ & - & - & - & - & - & - & - & - & - & - \\
\hline TB674 & $\mathrm{R}$ & $\mathrm{R}$ & $\mathrm{R}$ & - & - & - & - & - & - & - & - & - & - & - & - & - & - & MDR \\
\hline TB690 & $\mathrm{R}$ & $\mathrm{R}$ & $\mathrm{R}$ & - & - & - & - & - & - & - & - & - & - & - & - & - & - & MDR \\
\hline TB751 & $\mathrm{R}$ & - & - & - & - & $\mathrm{R}$ & - & - & - & - & - & - & - & - & - & - & - & - \\
\hline TB752 & $\mathrm{R}$ & $\mathrm{R}$ & $\mathrm{R}$ & $\mathrm{R}$ & - & - & - & - & - & - & - & - & - & - & - & - & - & MDR \\
\hline TB153 & $\mathrm{R}$ & $\mathrm{R}$ & $\mathrm{R}$ & - & - & - & - & - & - & - & - & - & $\mathrm{R}$ & - & - & - & - & MDR \\
\hline Total & $19 / 19$ & $14 / 19$ & $17 / 19$ & $5 / 19$ & $1 / 19$ & $4 / 19$ & $1 / 19$ & $1 / 19$ & 0 & 0 & 0 & 0 & $2 / 19$ & 0 & 0 & 0 & 0 & $14 / 19$ \\
\hline
\end{tabular}

Note: H: Isoniazid; E: Ethambutol; Z: Pyrazinamide; R: Rifampicin; S: Streptomycin; Km: Kanamycin; Am: Amikacin; Cm: Capreomycin; Ofx: Ofloxacin; Lfx: Levofloxacin; Mfx: Moxifloxacin; Eto: Ethionamide; Pto: Prothionamide; Cs: Cycloserine

PAS: Para aminosalicylate; Trd: Terizidone

$$
\begin{aligned}
\mathrm{R} & \text { : Resistance } \\
-\quad & \text { : No mutation in drugs resistance was found }
\end{aligned}
$$

There are differences between the results of geneXpert and NGS sample number TB618 and TB75. Both samples were resistant to rifampicin based on GeneXpert, but no mutations associated with rifampicin resistance are found on the results of the WGS (Table 1). The gene that codes for the formation of the RNA polymerase subunit $\beta$ enzyme is the rpoB gene. The enzyme is the target of rifampicin $(19,20)$. Fragments of the M. tuberculosis strain in the rpoB Papua strain in three samples, TB153, TB618 and TB751 are shown in Figure 1. Sample TB153 represents a sample that is resistant to Rifampicin based on geneXpert data and NGS data. The position of the mutation is indicated by red and yellow highlights (Figure 1A). Samples 618 and 751 did not have a mutation in the rpoB gene that could cause resistance to Rifampicin (Figures 1B and 1C).

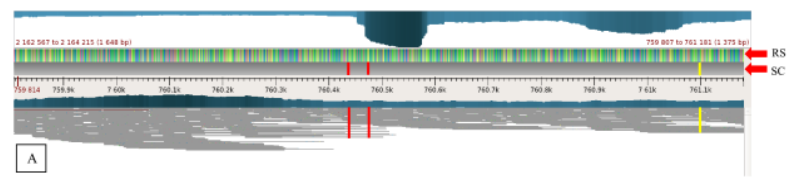

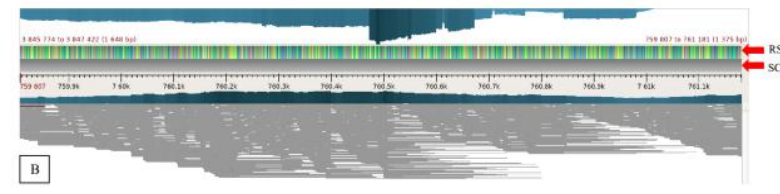

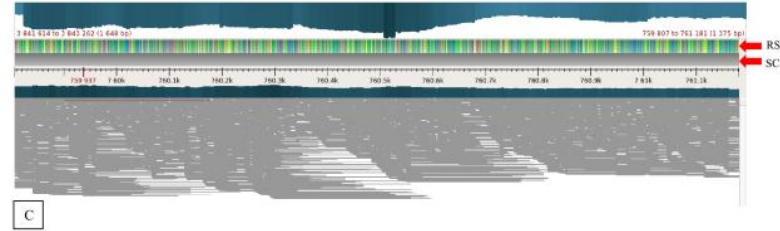

Note: RS= Reference Sequence (H37Rv (NC_000962.3)); $\mathrm{SC}=$ Sequence consensus

Figure 1. Comparison of the presence of mutations in the Papua $M$. tuberculosis $r p o B$ gene. 1A: sample 153 represents strains that are resistant to Rifampin both based on GeneXpert data and NGS data. The position of the mutation is indicated by red and yellow highlights. The gray area is the result of the alignment of the NGS reads. 1B and 1C: samples 618 and 715 are samples that do not have mutations in the M. tuberculosis strain rpoB Papua.

The quality of Papuan strain M. tuberculosis NGS results showed high confidence. Most of the mutations obtained occur in nucleotides 761155 M. tuberculosis. The mutation that occurs is the change in cytosine into thymine (Figure 2A). The quality of Papuan M. tuberculosis NGS results showed high confidence results. The scanned shoot region is a comparison of regions that contain mutations and do not contain mutations (Figures 2B and 2C) in the $M$. tuberculosis strain of rpoB Papua. 

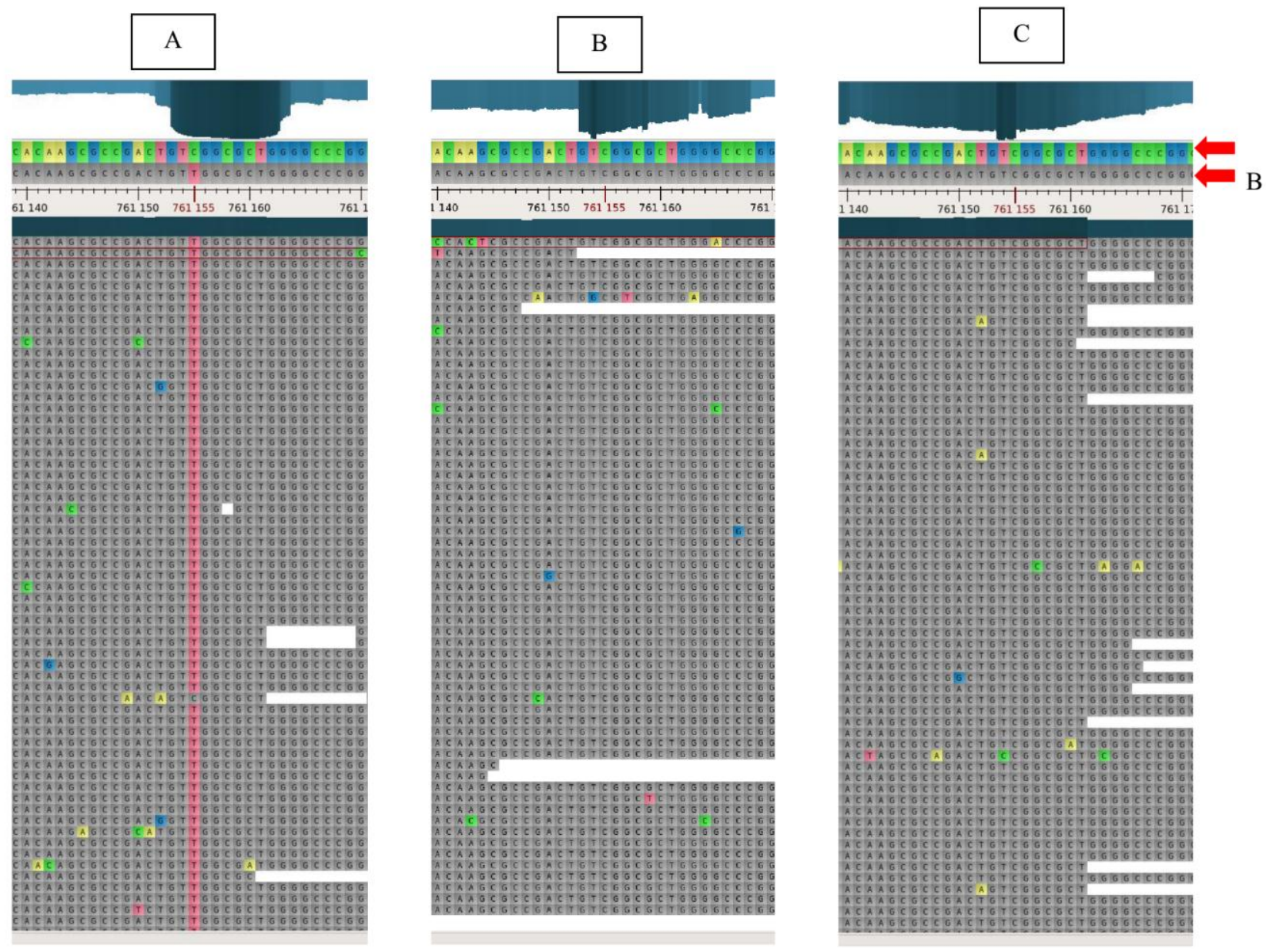

Figure 2. Comparison of mutation positions in the M. tuberculosis strain rpoB Papua. The quality of Papuan $M$. tuberculosis NGS results is shown by a large number of sequences that compose the $M$. tuberculosis genome. Figure $2 \mathrm{~A}$ is a sample of 153 containing mutations in the $r p o B$ gene. Figures $2 \mathrm{~B}$ and $2 \mathrm{C}$ are samples 618 and 751 which do not contain mutations in the $M$. tuberculosis rpoB gene.

\section{Discussion}

In this Papua strain M. tuberculosis study, WGS results show good data quality (Figure 1). The WGS results are very full power in obtaining the entire $M$. tuberculosis genome. WGS allows the examination of the whole genome of $M$. tuberculosis in mutations that provide drug resistance. Additionally mutations that occur outside of genes that are known to be related to drug resistance can be identified from the entire TB genome. ${ }^{9,10,15,16}$

In this paper, we focus on the drug resistance profile from the NGS data. Data analysis is performed using a bioinformatics approach. Specifically for the prediction of anti-tuberculosis drug resistance we used TB Profiler. TB profiler supplies the huge data of $M$. tuberculosis mutation that be compiled and reviewed from many TB studies around the world. The library consists of 1,325 mutations which are the most comprehensive and accurate data sources ever reported [17].

Based on TB Profile, prediction of resistance to antituberculosis drugs both in the first line and line 2 which are grouped into 5 groups (in this article only discussed 4 groups) (Table 1). Group 1 is a first-line drug consisting of Isoniazid, Ethambutol, Pyrazinamide, Rifampicin, Streptomycin. Group 2 Second-line injection drugs consist of Kanamycin, Amikacin, Capreomycin. Group 3 is a fluoroquinolone group consisting of Ofloxacin, Levofloxacin and Moxifloxacin. Group 4 consists of Ethionamide, Prothionamide, Cycloserine, Para aminosalicylates, Terizidone [2,21] Tagliani et al, using targeted next-generation sequencing on DNA extracted directly from sputum specimens. This allowed the detection of mutations associated with resistance to other first-line 
and second-line anti-TB drugs in addition to rifampicin without the need of obtaining pure MTB isolates [15,22].

A total of 14 samples were MDR TB (Table 1). MDR TB is a condition where $M$. tuberculosis is resistant to at least isoniazid (H) and rifampicin (R) [2]. In the first line, most of the samples were resistant to Isoniazid (14/19) and Rifampicin (17/19) while Ethambutol 5 samples, Pyrazinamide 1 samples and Streptomycin 4 samples. The most common mutation found in the Papua M. tuberculosis $r p o B$ gene is $\mathrm{S} 450 \mathrm{~L}$. This mutation is also most commonly found in other countries $[7,23]$.

In this study, samples are screened previously using geneXpert and detected as Rifampicin resistant. MDR $M$. tuberculosis can be detected rapidly on the geneXpert MTB/RIF system, but a rapid test for extensively drugresistant (XDR) cases is unavailable [15]. Unfortunately, geneXpert is lacked on sensitivity to detect the mutation in the non-target region and only good for detect rifampicin mutation. ${ }^{14,15,16}$ Interestingly, samples TB618 and TB751 were not resistant to rifampicin based on TBProfile (Table 1). Referring to the Papuan M. tuberculosis NGS data in the $r p o B$ gene (Figures $1 \mathrm{~B}$ and $1 \mathrm{C}$ ) it is known that there are no mutations in the $r p o B$ gene associated with rifampicin resistance. In another study, differences were found between the results of NGS and geneXpert where in samples that were sensitive to geneXpert, they were resistant to Rifampicin in the NGS and DST methods. ${ }^{9}$ False positives are also found in other studies comparing geneXpert MTB/RIF results, phenotypic methods and sequencing methods [24].

Besides differences results among several tests in the Rifampicin resistance test, Maningi, et al [11] reveal that there are differences between NGS and phenotypic Drugs Susceptibility Test (DST) base on the complete profile of mutations in the pncA gene from the NGS results. ${ }^{11}$ This is one of the powers of NGS assay that can be used to identify false-positive results. ${ }^{8}$ Information about antimicrobial resistance could be used in clinical decision making, although more data are needed on the correlation between genotype and phenotype before this can be used in clinical practice $[8,25]$.

In Group 2 there is one sample that is resistant to Kanamycin and Amikacin as a second-line drug (Sample TB618). The sample does not have mutations associated with isoniazid and rifampicin resistance, but is resistant to streptomycin. No resistance is found in group 3 whereas in group 4 there is one sample that was resistant to Ethionamide (Table 1). The resistance profile of this Papuan $M$. tuberculosis strain shows that some of the samples were MDR TB and that there were no XDR TB samples. Nevertheless, resistant samples have been found in the second line of OAT. This provides information on vigilance in treatment to avoid the emergence of XDR TB in Papua.

The results of this study provide a complete picture of the resistance profile of $M$. tuberculosis from Papua. In general, the WGS results reveal resistance to first-line and second- line TB drugs. Drug resistance profiling using nextgeneration sequencing offers rapid assessment of resistance-associated mutations, thus accelerating access to effective treatment $[4,26]$. The role of NGS in medical microbiology laboratories will increase during the next years, not only for research, but also, and more importantly, for molecular diagnostics, infection prevention, the investigation of outbreaks by the use of a unique outbreak marker approach, the characterization and surveillance of pathogens, the detection of novel resistance genes and for the application of a metagenomics approach on clinical samples [27]. Another advantage of using the NGS method is time effectiveness. Prediction of drug susceptibility and resistance using WGS such as diagnostic workflow with data generated in 9 days and at a price $7 \%$ cheaper, strong performance with high sensitivity and specificity to predict first-line drugs (rifampicin and isoniazid) resistant [14].

\section{CONCLUSION}

We conclude that Whole Genome Sequencing technology is the advanced technology that may apply to predict the resistance of TB drugs even MDR or XDR and distinguish the accurate drugs for patients individually.

\section{REFERENCES}

[1] E.M. Clarke, E.A. Emerson, Design and synthesis of synchronization skeletons using branching time temporal logic, in: D. Kozen (Eds.), Workshop on Logics of Programs, Lecture Notes in Computer Science, vol. 131, Springer, Berlin, Heidelberg, 1981, pp. 52-71. DOI: https://doi.org/10.1007/BFb0025774

[2] Kemenkes RI. Profil Kesehatan Indonesia 2018. Jakarta; 2019. 1-556 p. Available from: http://www.depkes.go.id/resources/download/pusdatin/ profil-kesehatan-indonesia/Profil-Kesehatan-Indonesia2016.pdf

[3] Kemenkes RI. Pedoman Nasional Pengendalian Tuberkolusis 2011-2014. 2011. Kemenkes: Jakarta

[4] WHO. The use of next-generation sequencing technologies for the detection of mutations associated with drug resistance in Mycobacterium tuberculosis complex: technical guide. Geneva; 2018. 15-128 p.

[5] Sotgiu G, Centis R, Migliori GB. Tuberculosis Treatment and Drug Regimens. Cold Spring Harb Perspect Med. 2015;5(a017822):1-12.

[6] Ford C, Yusim K, Ioerger T, Feng S, Chase M, Greene $\mathrm{M}$, et al. Mycobacterium tuberculosis e Heterogeneity revealed through whole genome sequencing. Tuberculosis. 2012;92(3):194-201. Available from: 
[7] Ssengooba W, Meehan CJ, Lukoye D, William G, Musisi K, Joloba ML, et al. Infection, Genetics and Evolution Whole genome sequencing to complement tuberculosis drug resistance surveys in Uganda. Infection, Genetics and Evolution. 2016;40:8-16. Available from: http://dx.doi.org/10.1016/j.meegid.2016.02.019

[8] Bouzouita I, Cabibbe AM, Trovato A, Daroui H, Ghariani A, Midouni B, et al. Whole-Genome Sequencing of Drug-Resistant. Emerg Infect Dis. 2019;25(3).

[9] Koser CU, Ellington MJ, Peacock SJ. Wholegenome sequencing to control antimicrobial resistance. Trens Genet. 2014;30(9):401-7.

[10] Daum L, Konstantynovska O, Solodiankin O, Liashenko O, Poteiko $\mathrm{P}$, Bolotin V, et al. NextGeneration Sequencing for Characterizing Drug Resistant Mycobacterium tuberculosis Genes from Clinical Isolates in the Ukraine. J Clin Microbiol. 2018;(March).

[11] Shea J, Halse TA, Lapierre P, Shudt M, Kohlerschmidt D, Roey P Van, et al. Comprehensive Whole-Genome Sequencing and Reporting of Drug Resistance Profiles on Clinical Cases of Mycobacterium tuberculosis in New York. J Clin Microbiol. 2017;55(6):1871-82.

[12] Maningi NE, Daum LT, Rodriguez JD, Mphahlele M, Peters RPH, Fischer GW, et al. Improved Detection by Next-Generation Sequencing of Pyrazinamide Resistance in Mycobacterium tuberculosis Isolates. J Clin Microbiol. 2015;53(12):3779-83.

[13] Gygli SM, Keller PM, Ballif M, Reinhard M, Ritter C, Sander P, et al. Whole-Genome Sequencing for Drug Resistance Profile Prediction in Mycobacterium tuberculosis. Antimicrob Agents Chemother. 2019;63(4):1-13.

[14] Omar S V., Joseph L, Said HM, Ismail F, Ismail N, Gwala TL, et al. Whole genome sequencing for drug resistance determination in Mycobacterium tuberculosis. Afr J Lab Med. 2019;8(1):1-5.

[15] Satta G, Lipman M, Smith GP, Arnold C, Kon OM, Mchugh TD. Mycobacterium tuberculosis and wholegenome sequencing: how close are we to unleashing its full potential? Clin Microbiol Infect. 2017;1-6. Available from: https://doi.org/10.1016/j.cmi.2017.10.030
[16] Brown AC, Bryant JM, Einer-jensen K, Holdstock J, Houniet DT, Chan JZM, et al. Rapid Whole-Genome Sequencing of Mycobacterium tuberculosis Isolates Directly from Clinical Samples. J Clin Microbiol. 2015;53(7):2230-7.

[17] Iketleng T, Lessells R, Dlamini MT, Mogashoa T, Mupfumi L, Moyo S, et al. Mycobacterium tuberculosis Next-Generation Whole Genome Sequencing : Opportunities and Challenges. Tuberc Res Treat. 2018;2018:1-8.

[18] Coll F, McNerney R, Preston MD, GuerraAssunção JA, Warry A, Hill-Cawthorne G, et al. Rapid determination of anti-tuberculosis drug resistance from whole-genome sequences. Genome Med. 2015;7(51):110 .

[19] Okonechnikov K, Golosova O, Fursov M, Varlamov A, Vaskin Y, Efremov I, et al. Unipro UGENE: A unified bioinformatics toolkit. Bioinformatics. 2012;28(8):1166-7.

[20] Feklistov A, Mekler V, Jiang Q, Westblade LF, Irschik H, Jansen R, et al. Rifamycins do not function by allosteric modulation of binding of $\mathrm{Mg} 2+$ to the RNA polymerase active center. Proc Natl Acad Sci. 2008;105(39):14820-5. Available from: http://www.pnas.org/cgi/doi/10.1073/pnas.0802822105

[21] McClure WR, Cech CL. On the Mechanism of Rifampicin Inhibition of RNA Synthesis. J Biol Chem. 1978;253(24):8949-56.

[22] WHO. Treatment of Tuberculosis Guidelines. Fourth Edi. Switzerland; 2010. 1-160 p.

[23] Tagliani E, Hassan MO, Waberi Y, Filippo MR De, Falzon D, Dean A, et al. Culture and Next-generation sequencing-based drug susceptibility testing unveil high levels of drug-resistant-TB in Djibouti : results from the first national survey. Sci Rep. 2017;(November):1-9. Available from: http://dx.doi.org/10.1038/s41598-01717705-3

[24] Dixit A, Freschi L, Vargas R, Calderon R, Sacchettini J, Drobniewski F, et al. Whole genome sequencing identifies bacterial factors affecting transmission of multidrug-resistant tuberculosis in a high-prevalence setting. Sci Rep. 2019;9(5602):1-10.

[25] Williamson DA, Basu I, Bower J, Freeman JT, Henderson G, Roberts SA. An evaluation of the Xpert MTB / RIF assay and detection of false-positive rifampicin resistance in Mycobacterium tuberculosis. Diagn Microbiol Infect Dis. 2012;74(2):207-9. 
Available from:
http://dx.doi.org/10.1016/j.diagmicrobio.2012.06.013

[26] Besser J, Carleton HA, Lindsey RL, Trees E. Nextgeneration sequencing technologies and their application to the study and control of bacterial infections. Clin Microbiol Infect. 2018;24(4):335-41. Available from: https://doi.org/10.1016/j.cmi.2017.10.013

[27] Phelan J, Sullivan DMO, Machado D, Ramos J, Whale AS, Grady JO, et al. The variability and reproducibility of whole genome sequencing technology for detecting resistance to anti-tuberculous drugs. Genome Med. 2016;8(132):1-9. Available from: http://dx.doi.org/10.1186/s13073-016-0385-x

[28] Deurenberg RH, Bathoorn E, Chlebowicz MA, Couto N, Ferdous M, García-cobos S, et al. Application of next generation sequencing in clinical microbiology and infection prevention. J Biotechnol. 2017;243:1624. Available

from: 